\title{
The Characterization of New and Next-generation Materials
}

\author{
Mingdong Cai and Jiann-Yang Hwang
}

The long-term challenge of materials research is to provide sustainable materials to fully conduct programmed performance and to successfully survive (or dissolve intentionally into) the in-service environment. The current energy and environment crisis largely raises the bar for designing new and next-generation materials; however, it also opens up opportunities for collaborative research. The high demand for next-generation materials such as high-temperature aerospace materials, bio-degradable polymers, bio-plastics, piezo polymeric material, biomimetrics, and smart materials requires inter-disciplinary breakthroughs in both materials synthesis and processing. In the meantime, specific methodology and facility has to be developed to quantitatively resolve materials microstructure and properties. This JOM topic reviews research into new nanoscale and environmentally friendly materials, and novel characterization techniques together with applicable demonstrations.

The paper by B. Li et al. investigates the chemical variation in stamp sands distributed along the Keweenaw Peninsula of Michigan. This research employs traditional x-ray diffraction (XRD) and SEM-EDS to observe morphology of the stamp sand sample and identify metal-rich areas. Quantitative chemical analysis is performed by the inductively coupled plasma mass spectrometer technique. The results show that the stamp sands are primarily basaltic and rich in copper. Further biological tests indicate the inhibited growth of $S$. aureus and A. niger in the object-sand containing solution, which leads the authors to believe that the stamp sand is both antibacterial and antifungal. Such research paves the path for transforming the waste of stamp sand into environmentally friendly production of antimicrobial roof granular.

The next two papers address two relatively new and versatile nondestructive characterization techniques-X-ray microCT and photoemission electron microscopy (PEEM). In the paper by C.L. Lin et al., cone beam $\mathrm{x}$-ray microCT is applied to quantify the three-dimensional porous structure in a gypsum wallboard. With improved algorithm and calibration, X-ray microCT can be used to characterize complex internal structure in a non-conductive materials structure. In the paper by G. Xiong et al., the principles and contrast mechanisms of PEEM are reviewed. Although extensive application in interrogating both static and dynamic electronic properties of complex nanostructured materials (including metals and semiconductors) has been reported, this paper highlights real-time in-situ examination of surface dynamics in a thermally induced structural phase transformation in barium titanate, inter-diffusion between thin metal copper and ruthenium layers, and multiphoton imaging of polystyrene nanoparticles on a silver-coated substrate. It is anticipated that novel characterization techniques, such as $\mathrm{x}$ ray micro $\mathrm{CT}$ and $\mathrm{PEEM}$, will be critical in understanding physics and explaining materials behaviors at different temporal and spatial scales.

As mentioned earlier, the search for alternative energy is always restricted by materials. The paper by K. Yasuda and T.H. Okabe reviews technologies to produce high-purity solar-grade silicon, focusing primarily on a process based on metallothermic reduction. Such research is critical to increase energy conversion rates and boost photovoltaic power generation. After a detailed description and literature review, the authors report that metallothermic reduction of silicon compounds can be cost-effective with high productivity, and that this technology is feasible for large-scale and high-speed production processes.

The paper by R. Varshney et al. explores a green technology for synthesizing copper nanoparticles using nonpathogenic bacterial strain Pseudomonas stutzeri. The bacterial strain was extracted from soil around a sewage outfall, and the synthesis was conducted by mixing the bacterial solution with copper sulfate and incubated at room temperature. The resultant spherical cobalt nanoparticles were determined to be stable and further characterization by XRD and quantified by high resolution transmission electron microscopy. It is noted that such an easy and costeffective technique is potentially useful in controlling metal ions in the aqueous environment.

\footnotetext{
Mingdong Cai is Senior Metallurgist with WymanGordon Forgings, Inc. in Houston, Texas and Jiann-Yang Hwang is Professor in the Department of Materials Science and Engineering, Michigan Technological University. Both are advisors to JOM from the Materials Characterization Committee of the Extraction \& Processing Division of TMS.
}

\section{Mingdong Cai and Jiann-Yang Hwang are TMS Members!}

To read more about them, turn to page 9. To join TMS, visit www.tms.org/Society/Membership.aspx. 